\title{
Oral Contributions
}

[MS30 - 02] Treatment of anharmonic motion in X-ray wavefunction refinement (XWR).

J. M. Bakk, ${ }^{\text {a }}$ D. Jayatilaka, ${ }^{b}$ Y.-S. Chen, ${ }^{c}$ S. Grabowsky $^{\mathrm{b}}$

${ }^{a}$ Faculty of Chemistry, The University of Warsaw, Poland

${ }^{b}$ School of Chemistry and Biochemistry, The University of Western Australia, Perth

${ }^{c}$ Center for Advanced Radiation Source, The University of Chicago, USA

E-mail: jbak@chem.uw.edu.pl

In Hirshfeld Atom Refinement [1] (HAR) asperical atomic scattering factors are derived directly from the molecular wavefunctioncalculated for a given crystal structure and used to refine geometrical parameters and atom motion. In the current study HAR including anharmonic motion treatment was performed for the first time. Anharmonic motion for chlorine and sulfur atoms were refined on an example of two related crystal structures, against high resolution and low temperature $(12 \mathrm{~K})$ synchrotron X-ray data sets (measured at 15-ID-B of the Advanced Photon Source, Argonne, USA). The results of the refinement and shape of obtained Probability Density Functions (PDF) were analyzed with respect to data resolution cutoff. The PDFs obtained from HAR were compared to the PDFs resulting from Hansen-Coppens multipolar modelling.[2]

Subsequently, X-ray constrained wavefunction (XCW) fitting [3] was performed for the crystal structures. X-ray Wavefunction Refinement [4] (XWR $=$ HAR + XCW fitting) is an alternative method used to obtain experimental electron density and only XWR enable to obtain experimental wavefunction (wavefunction fitted to experimental data). The XWR performed with and without treating of anharmonic motions against the experimental data sets were analyzed in terms of density maps, bond critical point and integrated atomic properties. Outcomes of the XWRs were compared to the results of 'static'
XWR performed against theoretical structure factors from periodic boundary calculations [5], as well as, to results of the theoretical calculations itself. Refinement of anharmonic motion of chlorine and sulfur atoms was possible even with data truncated to a resolution of $\mathrm{d}=0.45 \AA$. However, significant features in the experimental residual density indicating anharmonic motion of chlorine and sulfur atoms were seen only when analyses were performed against all high resolution data, up to a resolution of $\mathrm{d}=0.37 \AA$. All the PDFs obtained from HAR and multipolar models for the studied crystal structures are positively defined. However, shape of the PDFs differs substantially among the models (Figure 1). Ultra high resolution data seems to be very important for refining anharmonic motion properly.
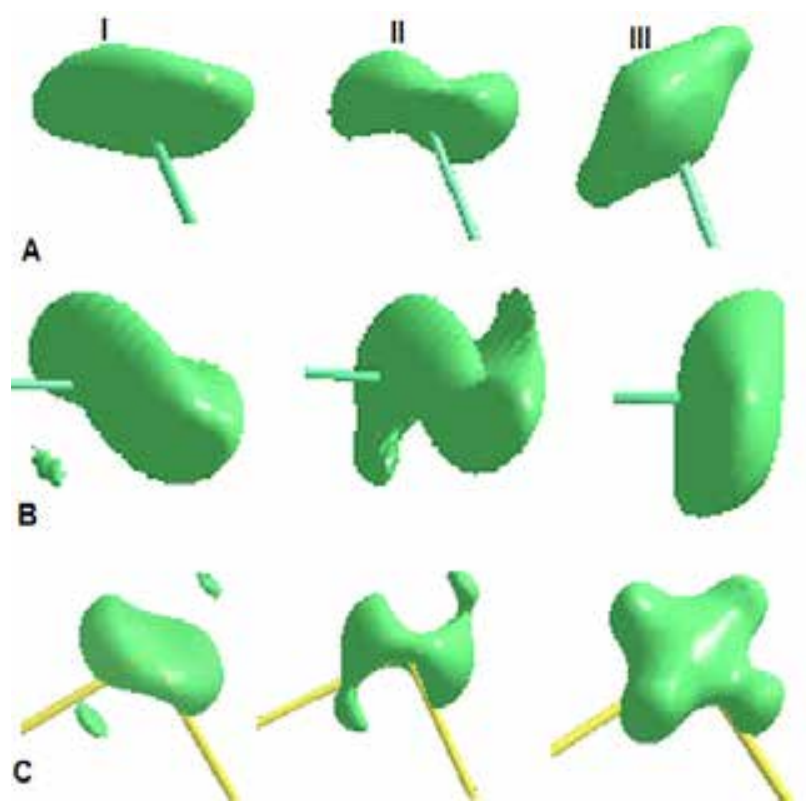

Figure 1 Representation of PDFs (50\% probability) obtained for A), B) chlorine atoms and C) sulphur atom of YQ28 crystal structure from different models: I) HAR performed against ultra high resolution data, II) HAR performed against data set truncated to a resolution of $\mathrm{d}=0.45 \AA$, III) multipolar model refined against ultra high resolution data. 
[1] D. Jayatilaka, B. Dittrich, (2008) Acta Cryst. A64 383-393.

[2] N. K. Hansen, P. Coppens, (1978) Acta Cryst. A34 909-921.

[3] D. Jayatilaka, D. Grimwood, (2001) Acta Cryst. A57 76-86.

[4] S. Grabowsky, D. Jayatilaka et al., (2012) Angew. Chem. Int. Ed. 51, 6776-6779.

[5] Dovesi, R., et al. (2008). CRYSTAL06 1.0. Version 1_0_2. University of Turin, Italy.

Keywords: Anharmonic motion; X-ray wavefunction refinement; experimental electron density 